\title{
Loading on rounded square foundation elements of offshore structures with marine growth at incidence
}

\author{
A. Frede ${ }^{1}$ and N. P. van Hinsberg ${ }^{1}$ \\ ${ }^{1}$ Institute of Aeroelasticity, German Aerospace Center, Göttingen, Lower Saxony, 37073, Germany
}

\begin{abstract}
A systematic wind tunnel study is performed to identify the mean and fluctuating loads experienced by a 2D square prism with non-dimensional rounded edges of $r / D=0.16$ and covered with hard marine fouling for Reynolds numbers between 70,000 and 8 million. The incidence angle is varied from $\alpha=-45^{\circ}$ to $3.25^{\circ}$ with increments of $3.25^{\circ}$ and $6.5^{\circ}$. The critical angle of incidence, at which a reattachment of the free shear layer takes place on the lateral windward directed side surface, is found at $\alpha_{c r}=-6.5^{\circ}$. For $\alpha<\alpha_{c r}$ two opposite trends - governed by the Reynolds number - of the mean drag coefficient, the r.m.s. of the lift fluctuations, and the Strouhal number are obtained. On the contrary, all aerodynamic parameters are relatively Reynolds-number independent for $\alpha>\alpha_{c r}$. For the latter angles of incidence, a decrease towards $-45^{\circ}$ induces higher values of $C_{D}$ and the r.m.s. of the lift fluctuations, as well as lower Strouhal numbers and mean lift forces.
\end{abstract}

\section{Keywords}

incidence; Reynolds number; prism; loading; separation

\section{Introduction}

The flow around prismatic and circular structures has received much attention in the last decades, motivated by the highly complex relationship between object shape, the flow behaviour around this type of bluff bodies, and the resultant fluid-structure interactions. Transition and separation of the surface boundary layer, free shear layer instability, flow reattachment, and the highly dynamic flow behaviour in the near and far wake are just a few of the many complex aerodynamic phenomena that take place and as a result of which the structure may undergo vortexinduced vibrations (VIV). Full and detailed knowledge of the unsteady aerodynamic loading on these kinds of structures, as well as of the process of vortex formation in the base region and the near- and far-wake topology as a function of the structure's cross-section and Reynolds number is essential to minimise the possible occurrence of VIV or even suppress it completely.

For circular cylinders it is well known that the behaviour of the flow around them is strongly dependent on the Reynolds number $R e_{D}=\frac{u_{\infty} D}{v}$, with the free-stream fluid velocity $u_{\infty}$, the cylinder's diameter $D$ and the kinematic viscosity of the fluid $v$. The locations of laminar-turbulent transition, boundary layer separation, and shear layer reattachment move along the surface with a change in Reynolds number, thereby inducing a strong influence on the overall aerodynamic loading $[10,11]$ and making them prone to VIV. On the contrary, the boundary layer separation points of prisms with square or rectangular cross-sections are fixed to their sharp leading edges and, beyond $\operatorname{Re}_{D}=10^{3}$, their aerodynamics is highly independent of the Reynolds number. However, both the flow topology around and the aerodynamic loading on these structures are now distinct functions of the angle of incidence $[3,4,7,8]$. A one-sided flow reattachment of the separated shear layer takes place above the critical angle of incidence of about $\pm 13^{\circ}[4,12]$ that leads to a clear asymmetric flow field around the prism and the appearance of a distinct steady lift force.
A combination of these two generic cross-sectional shapes, hence prisms with a squared cross-section having rounded edges, is nowadays more often used to optimise their fluidstructural behaviour, based on the positive flow characteristics of each of both generic cross-sections. Early experimental investigations by Delany and Sorensen [4] and Polhamus [9] on smooth squared prisms with rounded edges in cross-flow showed that an increase in the edge radius has a positive effect on the aerodynamic loading, as it lowers the drag force at equal Reynolds number on such prisms and shifts both the critical and supercritical flow states to lower Reynolds numbers. These observations were later confirmed through wind tunnel studies on smooth rounded square prisms by Tamura et al. [13], Carassale et al. [3], and van Hinsberg et al. [5], who also reported a decrease of the fluctuating lift coefficient and an increase in the vortex shedding frequency compared with their sharp-edged counterparts. They concluded that the rounded surface of the prism allows the movements of the boundary layer transition and primary separation points, as well as of the shear layer reattachment and secondary turbulent boundary layer separation points with Reynolds number similarly to circular cylinders, although for the former their movements are less pronounced.

Besides the amount of corner roundness, the effects of a variation in incidence angle on the aerodynamic behaviour of rounded square prisms at relatively high Reynolds numbers has been subject of several studies as well. Wind tunnel experiments by Tamura and Miyagi [12] and Carassale et al. [3] showed that the critical angle of incidence, being $13^{\circ}$ for sharpedged square prisms, decreases with increasing edge roundness as a result of the favourable promotion of the free shear layer reattachment on the lateral face exposed to the wind. Carassale et al. [3] furthermore showed that for their square prism with an edge roundness of $r / D=2 / 15$ an intermittent behaviour is present around this critical incidence angle in laminar flow and that for angles of incidence below $\alpha_{c r}$ an inversion of the lift slope appears in case of a turbulent flow. They concluded that the former phenomenon prohibits the use of the quasi-steady assumption to predict the galloping behaviour of this prism in laminar flow.

In addition to the object's cross-sectional shape, its angle of incidence and the Reynolds number, the surface texture of prismatic structures is also an important influencing parameter, in particular in marine engineering. Since submerged constructions are exposed to seawater, the natural process of marine growth induces a continuous increase of their surface roughness. The increased roughness height not only results in higher drag forces, but also increases both the vortex strength and their spanwise coherence - hence, giving rise to even higher periodic lift forces - and lowers at the same time the vortex shedding frequency, a combination that makes them more susceptible towards VIV [6]. The underlying physical mechanisms are a triggering of the transition and separation of the surface boundary layer, as well as of the shear layer reattachment at lower Reynolds numbers, thus a shift of the various flow phenomena, known for smooth prisms, to lower Reynolds numbers 
with increasing surface roughness $[1,6]$. This results in the appearance of the transcritical flow state, with its high drag and periodic lift forces and low Strouhal numbers, at relatively low Reynolds numbers.

All in all, the detailed prediction of the aero- and hydrodynamic behaviour of rounded square prisms with surface roughness in cross-flow is complex, but at the same time very challenging. Most studies currently available are limited to small angles of incidence and Reynolds numbers up to $10^{5}$. The influence of the incidence angle at realistic Reynolds numbers up to $10^{7}$ in combination with hard marine fouling is still not well understood. The current wind tunnel study aims at filling this gap by characterizing their effects on the mean and fluctuating lift and drag forces, the vortex shedding frequency, and the mean surface pressure distribution of a square prism with rounded edges of $r / D=0.16$ and a surface roughness height of $k_{s} / D=4.5 \times 10^{-4}$. Angles of incidence between $3.25^{\circ}$ and $-45^{\circ}$ and Reynolds numbers between $7 \times 10^{4}$ and $8 \times 10^{6}$ were investigated.

\section{Experimental test setup}

The experiments were performed in the High-Pressure Wind Tunnel Göttingen (DNW-HDG), a closed-circuit wind tunnel with a test section of $1 \mathrm{~m}$ in length and a cross-section of $0.6 \times 0.6 \mathrm{~m}^{2}$. The wind tunnel can be pressurised from atmospheric pressure up to $10 \mathrm{MPa}$. The free-stream velocity can be varied in steps of $0.1 \mathrm{~m} / \mathrm{s}$ from $4 \mathrm{~m} / \mathrm{s}$ up to $35 \mathrm{~m} / \mathrm{s}(\approx 0.1 \mathrm{Ma})$. The combination of a variation in pressure and velocity allows Reynolds numbers - based on the prism width $D$ - up to $10^{7}$, thereby covering the Reynolds number regimes from subcritical up to transcritical. A more detailed description of the flow facility can be found in van Hinsberg [5, 6].

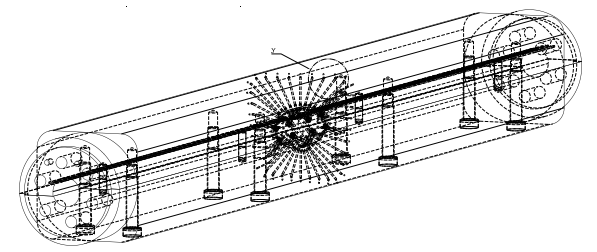

Figure 1. Technical drawing of the model with the pressure taps over the model's midspan.

The wind tunnel model is a $2 \mathrm{D}$ prism with a squared crosssection, a length of $L=600 \mathrm{~mm}$ and a side width of $D=60 \mathrm{~mm}$. The resulting aspect ratio is $L / D=10$. The prism edges are rounded to a dimensionless radius of $r / D=0.16$. The surface of the model is covered with a rough metal coating to produce a relative surface roughness of $k_{S} / D=(4.5 \pm 0.2) \times 10^{-4}$. At the mid-span section of the prism, 36 static pressure taps are equally distributed over the cross-section to obtain the mean surface pressure distribution. A schematic drawing of the model and it's cross-sectional shape are shown in the Figures 1 and 2, respectively.

The model is mounted horizontally in the test section. Both ends are connected to rigid piezoelectric balances to obtain the global (3D) unsteady lift and drag forces. Power spectra of the lift fluctuations are thereupon computed to determine the main vortex shedding frequency. The mean surface pressure distribution is not only used to obtain the $2 \mathrm{D}$ mean aerodynamic force and pitch moment coefficients, but also to extract information on the positions of the boundary layer primary and secondary separation and the reattachment locations.

In this experiment a Reynolds number range from $7 \times 10^{4}$ up

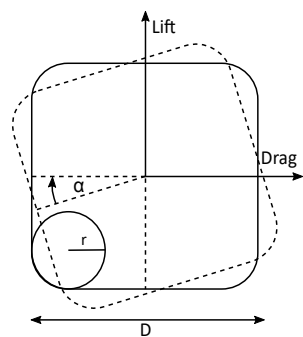

Figure 2. Schematic drawing of the prism cross-section with the definitions of the aerodynamic load vectors, edge radius and incidence angle.

to $8 \times 10^{6}$ was covered. For each angle of incidence the influence of the Reynolds number was studied by changing the freestream velocity from $4 \mathrm{~m} / \mathrm{s}$ up to $34 \mathrm{~m} / \mathrm{s}$ in steps of $1 \mathrm{~m} / \mathrm{s}$ at five different pressure values between $0.35 \mathrm{MPa}$ and $8 \mathrm{MPa}$. The angle of incidence was set by rotating the prism in a counterclockwise direction along its central axis, hence mostly negative angles of incidence between $3.25^{\circ}$ and $-45^{\circ}$ were investigated. For each combination of incidence angle and Reynolds number the global unsteady aerodynamic forces and the 2D static surface pressures were recorded over a period of $30 \mathrm{~s}$, the former at a sampling rate of $5 \mathrm{kHz}$. The wind tunnel blockage ratio of the prism lies between $0.1\left(\alpha=0^{\circ}\right)$ and $0.14\left(\alpha=-45^{\circ}\right)$. The free-stream velocity and the drag coefficient were thereupon corrected with the formulas by Allen and Vincenti [2], whereas the correction by Roshko [10] was applied to correct the mean surface pressure coefficients.

\section{Experimental results}

The 3D mean drag and lift coefficients are defined as $C_{D}=\overline{D(t)} / q_{\infty} D L$ and $C_{L}=\overline{L(t)} / q_{\infty} D L$, respectively, with $\overline{D(t)}$ and $\overline{L(t)}$ the mean drag and lift forces, $q_{\infty}$ the dynamic pressure and $L$ and $D$ the dimensions of the prism. The Strouhal number is calculated as $S t_{L}=f_{L} D / u_{\infty}$ with $f_{L}$ the main vortex shedding frequency in the PSD based on the time series of the lift force.

\section{Drag coefficient}

The change of $C_{D}$ with Reynolds number is shown in Figure 3 for five incidence angles between $0^{\circ}$ and $-45^{\circ}$. For $\alpha=0^{\circ}$ the drag coefficient is about 1.3 in the subcritical regime. The following critical regime is marked by a strong decrease in the drag coefficient, the so-called "drag crisis", of about $50 \%$. Its end is defined by the lowest value of the drag coefficient of $C_{D}$ $\approx 0.6$ at the critical Reynolds number of $R e_{c r}=4.22 \times 10^{5}$. Although a further increase in $R e$ up to $10^{7}$ induces small changes in the drag coefficient, the flow state remains supercritical.

A decrease in $\alpha$ to $-6.5^{\circ}$ leads to a lowering of the subcritical

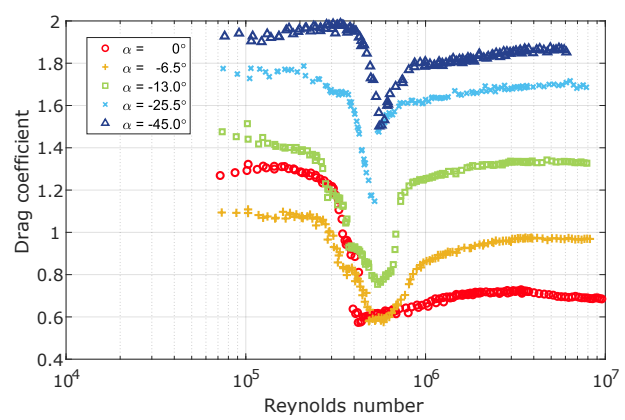

Figure 3. Overall mean drag coefficient $C_{D}$ as a function of the Reynolds number for various angles of incidence between $0^{\circ}$ and $-45^{\circ}$. 
drag coefficient. The drop of $C_{D}$ within the drag crisis is somewhat lower, as a result of which an equal minimum drag coefficient is reached at the end of the critical flow state as was obtained for $\alpha=0^{\circ}$, albeit at a somewhat higher Reynolds number of $R e=4.84 \times 10^{5}$. A limited supercritical flow state with low values of $C_{D}$ is now present up to $R e=6.23 \times 10^{5}$. For higher $R e$ a clear increase in $C_{D}$ is observed, followed by a constant drag coefficient of $C_{D}=1$ within the transcritical flow state.

For angles of incidence in the range of $-13^{\circ}$ to $-45^{\circ}$ the trends of the $C_{D}(R e)$-curves are similar to $\alpha=-6.5^{\circ}$. With decreasing $\alpha$ higher drag coefficients are obtained for all Reynolds numbers. Between $\alpha=-13^{\circ}$ and $-45^{\circ}$ the drag crisis decreases by about $50 \%$, whereas the supercritical flow state reduces to a single point and is now positioned at approximately equal Reynolds number for all angles of incidence. The drag recovery in the upper transition leads for all shown incidence angles to transcritical drag coefficients that are slightly lower than their subcritical counterparts.

\section{Strouhal number}

The dependency of the Strouhal number on the Reynolds number is shown in Figure 4. At $\alpha=0^{\circ}$ constant subcritical Strouhal numbers of about 0.135 are obtained, whereas slightly decreasing values with increasing Reynolds numbers are observed in the critical regime. At the critical Reynolds number, at which $C_{D, \text { min }}$ is reached, the Strouhal number jumps to $S t_{L}=0.3$, hence more than twice its subcritical value. At low supercritical Reynolds numbers, a steady decrease in $S t$ of about $17 \%$ occurs, coupled with a slight increase in $C_{D}$ in Figure 3, after which the Strouhal number remains at a relatively constant plateau for $R e>2 \times 10^{6}$. For $\alpha=-6.5^{\circ}$ a similar behaviour of the Strouhal number with the Reynolds number is found: constant subcritical values of $S t_{L}=0.174$, a slight decrease followed by a jump in $S t_{L}$ in the critical flow state and high supercritical Strouhal numbers of about 0.28. An additional steep drop in $S t_{L}$ from the super- to the transcritical flow state is observed at about $R e=8 \times 10^{5}$, followed by constant values up to the highest Reynolds number.

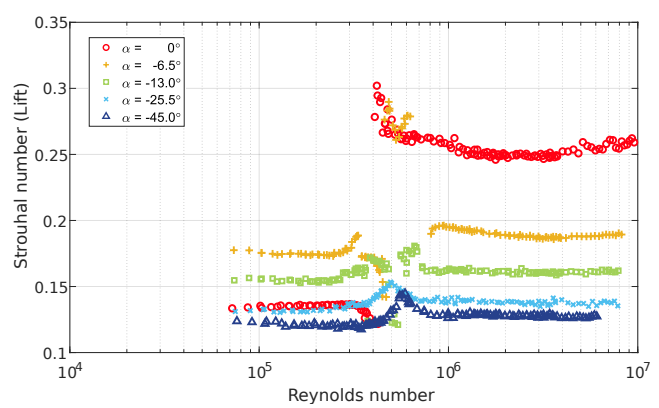

Figure 4. Reynolds-number dependency of the Strouhal number $S t_{L}$ at various angles of incidence between $0^{\circ}$ and $-45^{\circ}$.

With a further decrease in the angle of incidence, an increasing Reynolds-number independency of the Strouhal number can be observed. For the largest investigated angle of incidence of $-45^{\circ}$ the Strouhal number shows a slight dependency on the Reynolds number in the critical and upper transition flow states only. The increase in the Strouhal number in the critical flow state is merely $25 \%$ and also the decrease in the upper transition towards transcritical values is small in comparison with the results for $0^{\circ}$ and $-6.5^{\circ}$. At $\alpha=-45^{\circ}$ the jumps in $S t_{L}$ in both flow states have disappeared completely.

\section{Influence of the angle of incidence}

Figure 5 presents the effect of the angle of incidence on the global mean lift and drag coefficients, the Strouhal number, and the r.m.s. values of the fluctuating lift. For all four aerodynamic parameters no significant influence of the Reynolds number can be observed for angles of incidence between $-45^{\circ}$ and $-6.5^{\circ}$. Within this range of incidence angles a small scattering in the values of $C_{D}$ and of the r.m.s. of the lift fluctuations with $R e_{D}$ is seen to occur at constant angles of incidence, which becomes for both parameters quite significant at $\alpha=-6.5$. Whereas a decrease in angle of incidence leads on the one hand to a steady reduction of both the global mean drag coefficient and the r.m.s.
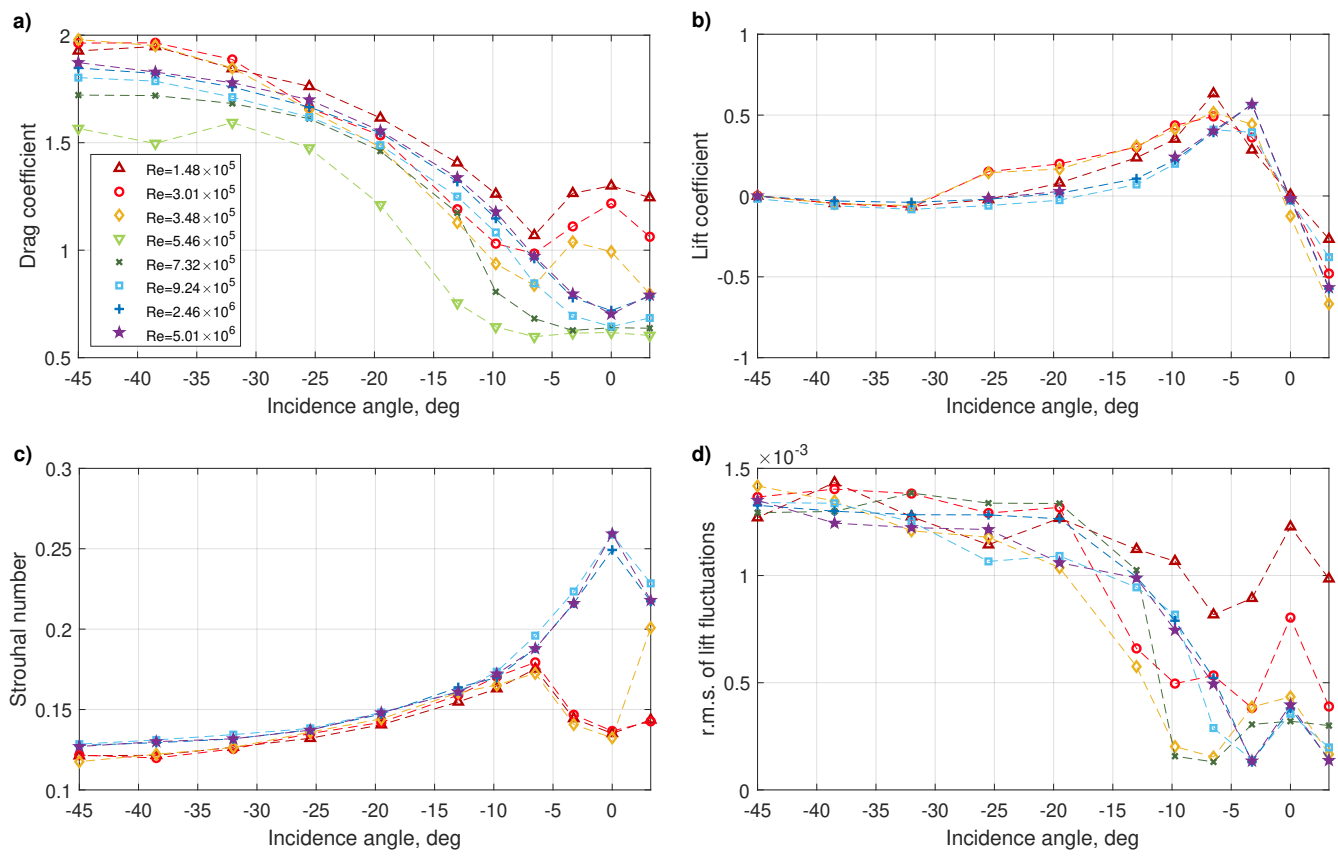

Figure 5. The overall mean drag coefficient $C_{D}$ (a), overall mean lift coefficient $C_{L}$ (b), Strouhal number $S t_{L}$ (c), and r.m.s. of the lift fluctuations $\sqrt{\overline{C_{L}^{2}}}$ (d) as function of the angle of incidence $\alpha$ for various Reynolds numbers between $1.48 \times 10^{5}$ and $5.01 \times 10^{6}$. 
of the lift fluctuations, it induces, on the other hand, an increase in the global mean lift coefficient and the Strouhal number. Figure 5a clearly shows that for these angles of incidence the lowest drag coefficients are obtained at $R e=5.46 \times 10^{5}$, hence within the critical flow regime.

The $C_{L}$-curves in Figure $5 \mathrm{~b}$ possess an infliction point at $\alpha=$ $-6.5^{\circ}$, except for $R e=5.01 \times 10^{6}$ for which the infliction is located at $-3.25^{\circ}$. At these critical incidence angles an inversion of the lift slope $\mathrm{d} C_{L} / \mathrm{d} \alpha$ from positive to negative takes place. A similar behaviour was reported by Carassale et al. [3] for square prisms with edge radii of $r / D=0,1 / 15$ and $2 / 15$ in smooth (turbulence intensity of $0.2 \%$ ) and turbulent (turbulence intensity of $6 \%$ ) flow. For their prism with an edge radius of $r / D=2 / 15$ they found a critical angle of $-5^{\circ}$. Although a somewhat coarser discretisation in incidence angle and a higher surface roughness value were selected in the current study, the measured critical angle of incidence of about $-6.5^{\circ}$ for the rounded slightly rough square prism with $r / D=0.16$ is consistent with the results of Carassale et al. [3].

For angles of incidence larger than $-6.5^{\circ}$ a change in the Reynolds-number-dependent behaviour of $C_{D}, S t_{L}$, and the r.m.s. of the lift fluctuations can be observed, as the trends of the curves become dependent on the critical Reynolds number $R e_{c r}$. For Reynolds numbers above this critical value both the global mean drag coefficient and the Strouhal number continue their trends with increasing angle of incidence, hence a steady decrease of the former and a steady increase of the latter up to their minimum and maximum values, respectively, at $\alpha=0^{\circ}$. On the contrary, for $R e<R e_{c r}$, minimum and maximum values of $C_{D}$ and $S t_{L}$, respectively, are reached already at $\alpha_{c r}=-6.5^{\circ}$. Just as it was described above for the global man lift coefficient, an inversion of the global mean drag and the Strouhal number slopes, $\mathrm{d} C_{D} / \mathrm{d} \alpha$ and $\mathrm{d} S t / \mathrm{d} \alpha$, from negative to positive and from positive to negative, respectively, take place. Absolute maximum values of $C_{D}$ and local minimum Strouhal numbers are now reached at $\alpha=0^{\circ}$. Although a large scattering among the values of the r.m.s. of the lift fluctuations between the various Reynolds numbers at constant angles of incidence is obtained, a similar behaviour with a change of the slope around $\alpha_{c r}$ and local maximum values at $\alpha=0^{\circ}$ can be observed.

\section{Conclusion}

The current wind tunnel study focused on the combined effects of incidence angle and Reynolds number on the aerodynamic behaviour of a squared prism with rounded edges of $r / D=0.16$ and a surface roughness of $k_{s} / D=4.5 \times 10^{-4}$ in cross-flow. A critical angle of incidence was found at $\alpha=-6.5^{\circ}$, for which a reattachment of the free shear layer takes place on the lateral face exposed to the wind. For $\alpha>\alpha_{c r}$ no large variations of the various aerodynamic parameters with Reynolds number were seen to occur. At the critical angle of incidence, inversions of the slopes of the global mean force coefficients, the r.m.s. of the lift forces, and the Strouhal number with incidence angle were found for Reynolds numbers smaller than the critical Reynolds number. This latter phenomenon led to two opposite trends of $C_{D}, S t_{L}$, and the r.m.s. of the lift fluctuations at angles of incidence smaller than $\alpha_{c r}$.

\section{Acknowledgements}

Technical assistance of Markus Löhr and Karsten Steiner of DNW (German Dutch Wind tunnels) is gratefully acknowledged. Systemhaus Technik and Deharde are recognised for the construction and manufacturing of the wind tunnel models. Finally, the authors would like to thank the reviewer for the fruitful comments.

\section{References}

[1] Achenbach, E. (1971). Influence of surface roughness on the cross-flow around a circular cylinder. Journal of Fluid Mechanics, 46(2), 321-335 (DOI:10.1017/s0022112071000569).

[2] Allen, M. J., and Vincenti, W. G. (1944). Wall interference in a tow-dimensional flow wind tunnel with consideration of the effect of compressibility. Nat. Adv. Comm. Aero. Technical Note, 782, 155-184.

[3] Carassale, L., Freda, A., and Marrè-Brunenghi, M. (2014). Experimental investigation on the aerodynamic behavior of square cylinders with rounded corners. Journal of Fluids and Structures, 44, 195-204 (DOI:10.1016/j.jfluidstructs.2013.10.010).

[4] Delany, N. K., and Sorensen, N. E. (1953). Low-speed drag of cylinders of various shapes. Nat. Adv. Comm. Aero. Technical Note, 3038, 1-22.

[5] Huang, R. F., Lin, B. H., and Yen, S. C. (2010). Timeaveraged topological flow patterns and their influence on vortex shedding of a square cylinder in crossflow at incidence. Journal of Fluids and Structures, 26(3), 406-429 (DOI:10.1016/j.jfluidstructs.2010.01.003).

[6] Okajima, A. (1982). Strouhal numbers of rectangular cylinders. Journal of Fluid Mechanics, 123, 379-398 (DOI:10.1017/S0022112082003115).

[7] Polhamus, E. C. (1958). Effect of flow incidence and Reynolds number on low-speed aerodynamic characteristics of several noncircular cylinders with applications to directional stability and spinning. Nat. Adv. Comm. Aero. Technical Note, 4176, 1-54.

[8] Roshko, A. (1961). Experiments on the flow past a circular cylinder at very high Reynolds number. Journal of Fluid Mechanics, 10(3), 345-356 (DOI:10.1017/s0022112061000950).

[9] Schewe, G. (1983). On the structure and resolution of wallpressure fluctuations associated with turbulent boundarylayer flow. Journal of Fluid Mechanics, 134, 311-328 (DOI:10.1017/s0022112083003389).

[10] Tamura, T., and Miyagi, T. (1999). The effect of turbulence on aerodynamic forces on a square cylinder with various corner shapes. Journal of Wind Engineering and Industrial Aerodynamics, 83, 135-145 (DOI:10.1016/S01676105(99)00067-7).

[11] Tamura, T., Miyagi, T., and Kitagishi, T. (1998). Numerical prediction of unsteady pressures on a square cylinder with various corner shapes. Journal of Wind Engineering and Industrial Aerodynamics, 74, 531-542 (DOI:10.1016/s0167-6105(98)00048-8).

[12] van Hinsberg, N. P. (2017). Experiments on the aerodynamic behaviour of square cylinders with rounded corners at Reynolds numbers up to 12 million Journal of Fluids and Structures, 74, 241-233 (DOI:10.1016/j.jfluidstructs.2017.08.002).

[13] van Hinsberg, N. P., Schewe, G., and Jacobs, M. (2018). Experimental investigation on the combined effects of surface roughness and corner radius for square cylinders at high Reynolds numbers up to $10^{7}$. Journal of Wind Engineering and Industrial Aerodynamics, 173, 1427 (DOI:10.1016/j.jweia.2017.12.003). 